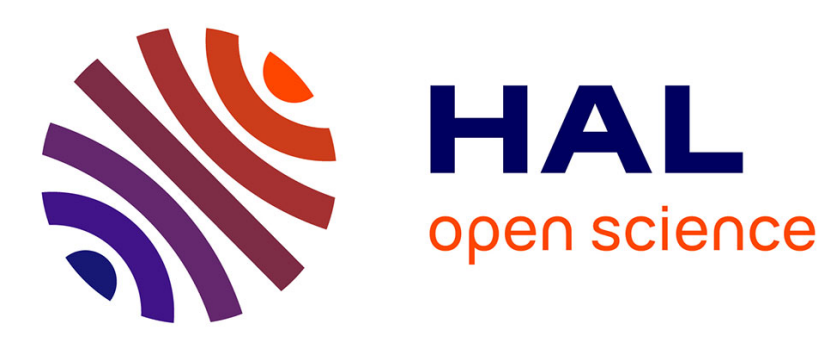

\title{
Theoretical study of the generation of soap films: role of interfacial visco-elasticity
}

Jacopo Seiwert, Benjamin Dollet, Isabelle Cantat

\section{To cite this version:}

Jacopo Seiwert, Benjamin Dollet, Isabelle Cantat. Theoretical study of the generation of soap films: role of interfacial visco-elasticity. Journal of Fluid Mechanics, 2014, 739, pp.124-142. $10.1017 / \mathrm{jfm} .2013 .625$. hal-00944297

\section{HAL Id: hal-00944297 https://hal.science/hal-00944297}

Submitted on 13 Feb 2014

HAL is a multi-disciplinary open access archive for the deposit and dissemination of scientific research documents, whether they are published or not. The documents may come from teaching and research institutions in France or abroad, or from public or private research centers.
L'archive ouverte pluridisciplinaire HAL, est destinée au dépôt et à la diffusion de documents scientifiques de niveau recherche, publiés ou non, émanant des établissements d'enseignement et de recherche français ou étrangers, des laboratoires publics ou privés. 


\title{
Theoretical study of the generation of soap films: role of interfacial visco-elasticity
}

\author{
Jacopo Seiwert, Benjamin Dollet and Isabelle Cantat $\dagger$ \\ Institut de Physique de Rennes, UMR 6251 CNRS/Université de Rennes 1, Campus Beaulieu, Bâtiment \\ 11A, 35042 Rennes CEDEX, France
}

(Received 18 July 2013; revised 9 November 2013; accepted 20 November 2013; first published online 17 December 2013)

In this work, we study theoretically the thickness of a liquid film (typically made of a surfactant solution) pulled out of a bath at constant speed in the absence of gravity, when it features a viscous or an elastic interfacial rheology. We show that a purely viscous rheology does not lead to the extraction of a steady state film of constant thickness. In contrast, the thickness of the film is well defined in the elastic case, which allows us to compute it. This thickness depends on the capillary number of the experiment, and on the elasticity of the interface. It is always lower than or equal to that obtained for an incompressible interface predicted by Frankel (Mysels, Shinoda and Frankel, Soap Films: Studies of their Thinning and a Bibliography, 1959), which is recovered in the limit of an arbitrary large elasticity.

Key words: foams, interfacial flows (free surface), thin films

\section{Introduction}

The formation of soap films from a solution reservoir is a key process of foam formation and foam shearing. Since the seminal work by Mysels, Shinoda \& Frankel (1959), numerous experimental and theoretical studies have focused on soap film entrainment by a solid frame (Lal \& di Meglio 1994; de Gennes 2001; Berg, Adelizzi \& Troian 2005; van Nierop, Scheid \& Stone 2008; Saulnier et al. 2011). The key theoretical prediction for the thickness $h_{F r}$ of the film that is created in such a geometry is Frankel's law (Mysels et al. 1959), $h_{F r}=2.68 r_{m} \mathrm{Ca}^{2 / 3}$, where $r_{m}$ and $\mathrm{Ca}$ are respectively the radius of curvature of the meniscus connecting the film to the bath, and the capillary number of the experiment, $C a=\eta U / \gamma$, with $\eta$ the viscosity of the solution, $U$ the withdrawal velocity and $\gamma$ the surface tension. This theory is in reasonable agreement with experimental data: in particular, the thickness scaling in $\mathrm{Ca}^{2 / 3}$ is well verified in most published experiments (see van Nierop et al. 2008 for a review). However, the absolute thicknesses vary significantly from this prediction, up to a factor of 2 .

The most important assumption made by Frankel is the incompressibility of the interfaces: his theory assumes a uniform velocity for the surfactant layer, equal to that of the solid frame. Although the details of the processes leading to this incompressibility are not known, satisfying this condition requires interfacial stresses (which may be computed a posteriori in Frankel's framework, once the velocity field 
in the film has been determined: see Cantat 2013). From a physical standpoint, these stresses arise from the stretching of the surfactant-covered interfaces. The relation between stresses and interfacial stretch is modelled with an interfacial extensional viscoelasticity, which may in practice be dominated either by elastic or by viscous effects. Frankel's theory explores the regime where the resulting deformations are negligible: the interfacial rheology becomes irrelevant, and a simple incompressibility relation suffices to close the problem.

The aim of this paper is to investigate theoretically the effect of a finite interfacial viscosity or elasticity (and thus finite interfacial stretches) on the thickness of the extracted soap film, in order to determine whether taking into account these effects could explain the discrepancies between Frankel's law and experiments. Using the classical framework of the lubrication approximation, we show that elastic interfaces lead to a steady solution with a film thickness smaller than or equal to that predicted by Frankel. The corresponding velocity field is a Poiseuille flow, with some elongational contribution. The steady film thickness only depends on a single non-dimensional parameter, involving the capillary number and the ratio between the surface elasticity $E$ and the surface tension $\gamma$. A Frankel's film is obtained at low $C a$, whereas a thinner film is obtained at higher $C a$. The critical capillary number between the two regimes varies as $C a^{c} \sim(E / \gamma)^{3 / 2}$, as deduced by scaling analysis in Lal \& di Meglio (1994). Our results are in qualitative agreement with experimental results obtained by Cohen-Addad \& di Meglio (1994), Lal \& di Meglio (1994) and Saulnier et al. (2011). However, a quantitative comparison would require a precise knowledge of the elastic modulus and its dependence on surfactant concentration, for the time and length scales relevant to the experimental situation and in the nonlinear regime, which is still an experimental and theoretical challenge (Sagis 2011; Alvarez, Walker \& Anna 2012).

In contrast, we show that a solution with purely viscous interfaces cannot form a steady film. Lastly, we consider the validity of elongational flow (plug flow) solutions, which cannot be excluded a priori, and which are considered, for example, for soap film in unsteady drainage in Howell \& Stone (2005). Following the method used in van Nierop et al. (2008) we show that the slightly different set of equations governing the flow in this case does not admit a steady solution for purely viscous interfaces. This is consistent with what is observed for viscous sheets or viscous threads, which continuously thin when pulled out of a bath (Wilson 1988; Gaudet, McKinley \& Stone 1996). We also investigate the case of elongational flow with elastic interfaces, which does not admit a solution either.

\section{Governing equations}

\subsection{Hydrodynamical problem}

We consider the problem sketched in figure 1: a film is pulled out of a meniscus of a surfactant solution, which acts as an infinite reservoir, at a constant velocity $U$ in the vertical direction $\boldsymbol{e}_{x}$. We assume invariance in the $z$-direction. In the geometry depicted in figure 1, the meniscus radius of curvature at the contact point with the film is $r_{m}=l_{c} / \sqrt{2}$, with $l_{c}=\sqrt{\gamma /(\rho g)}$ the capillary length, $g$ the gravity constant and $\rho$ the solution density. More generally, the solution reservoir can be any meniscus, for example a Plateau border in a foam (Seiwert et al. 2013), for which the radius $r_{m}$ is determined by the foam's liquid fraction.

In the following, we neglect inertial effects, as well as gravity, compared to the capillary forces. The first assumption imposes $\rho U^{2} \ll \gamma / r_{m}$, i.e. $W e=\rho U^{2} r_{m} / \gamma \ll$ 


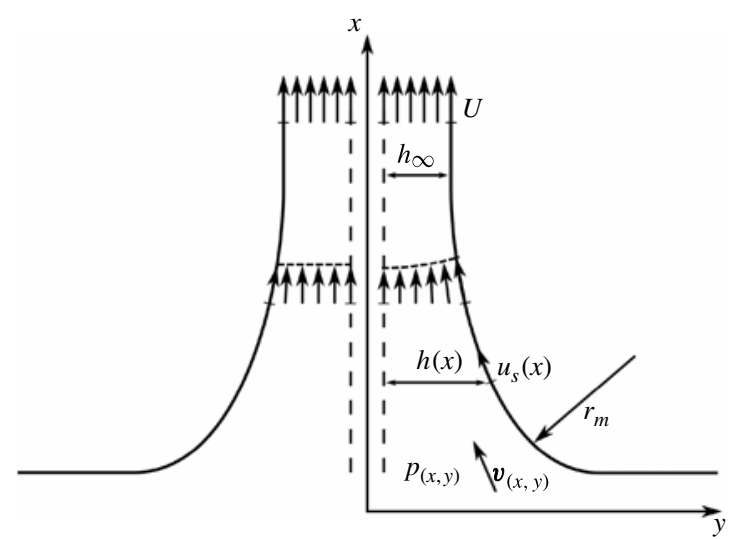

FIGURE 1. Sketch of the film and notations used in the text. A Poiseuille-type flow is represented on the right side and an elongational one on the left side. The dashed line is the symmetry axis, at the position $y=0$.

1, where $W e$ is the Weber number (Quéré 1999). This may be rewritten as $C a^{2} \ll \eta^{2} /\left(\rho \gamma r_{m}\right)$. For usual foaming solutions, $\rho \sim 10^{3} \mathrm{~kg} \mathrm{~m}^{-3}, \gamma \sim 3 \times 10^{-2} \mathrm{~kg} \mathrm{~s}^{-2}$, $\eta \sim 10^{-3} \mathrm{~kg} \mathrm{~s}^{-1} \mathrm{~m}^{-1}$ and, if $r_{m} \sim 10^{-3} \mathrm{~m}$, the upper boundary for $C a$ thus becomes $C a \ll 5 \times 10^{-3}$. Similarly, a negligible gravity imposes $C a \ll 1$ for films extracted from a meniscus whose typical curvature is the capillary length (Quéré 1999), which is thus less restrictive in our case.

We also impose a steady state: at large $x$, the film thickness tends towards a constant and uniform value $2 h_{\infty}$ and the velocity field tends to a plug flow at the imposed velocity $U$.

The liquid flow in the film is governed by the Stokes equations:

$$
\begin{gathered}
\eta \Delta v-\nabla p=0, \\
\operatorname{div} v=0,
\end{gathered}
$$

with $\boldsymbol{v}(x, y)=v_{x} \boldsymbol{e}_{x}+v_{y} \boldsymbol{e}_{y}$ the fluid velocity and $p$ its pressure.

The problem is symmetric with respect to the $y=0$ plane. Thus, $v_{y}(x, y=0)=0$ and $\partial_{y} v_{x}(x, y=0)=0$. The liquid/gas interface is defined by $y= \pm h(x)$, so the film thickness is $2 h(x)$. Along this surface, the continuity of normal and tangential stresses imposes

$$
\begin{aligned}
\boldsymbol{n} \cdot \boldsymbol{\tau} \cdot \boldsymbol{n} & =\boldsymbol{n} \cdot \nabla_{s} \boldsymbol{\tau}_{s} \\
\boldsymbol{t} \cdot \boldsymbol{\tau} \cdot \boldsymbol{n} & =\boldsymbol{t} \cdot \nabla_{s} \boldsymbol{\tau}_{s}
\end{aligned}
$$

where $\boldsymbol{t}$ and $\boldsymbol{n}$ are the tangent and the normal vectors of the interface, respectively, $\tau_{i j}=\eta\left(\partial_{x_{i}} v_{j}+\partial_{x_{j}} v_{i}\right)-p \delta_{i j}$ is the usual viscous stress tensor in the Newtonian liquid bulk, $\nabla_{s}=\boldsymbol{I}_{s} \cdot \boldsymbol{\nabla}$ is the surface gradient operator and $\boldsymbol{\tau}_{s}$ is the interfacial stress. The latter arises from both the surface tension and the viscosity of the surfactant layer. In two-dimensional problems, its general expression for Newtonian interfaces is $\boldsymbol{\tau}_{s}=\left(\gamma+\eta_{s}^{*} \boldsymbol{I}_{s}: \boldsymbol{D}_{s}\right) \boldsymbol{I}_{s}=\tau_{s} \boldsymbol{I}_{s}$. Here, the surface tension $\gamma(x, t)$ is a dynamical variable of our problem, whose properties (and relation to interface deformations) are discussed below; $\eta_{s}^{*}$ is the interfacial viscosity of the film $\left(\eta_{s}^{*}=\eta_{s}+\kappa_{s}\right.$, with $\eta_{s}$ and $\kappa_{s}$ the shear and dilatational interfacial viscosities, as discussed in appendix A), $\boldsymbol{I}_{s}$ is the surface projection tensor and $\boldsymbol{D}_{s}$ is the velocity gradient tensor at the interface. 
Finally, mass conservation imposes, at steady state, a constant flux (in the half-film) $q=U h_{\infty}$

\subsection{Interfacial elasticity}

To close the problem, the surface tension $\gamma$ needs to be related to the other parameters. Strictly speaking, $\gamma$ is a function of the local concentration of surfactant adsorbed on the interface, $\Gamma$. In principle, the value of $\Gamma$ could be obtained by explicitly taking into account surfactant transport: diffusion and convection in the bulk and within the interface, and exchange dynamics between the bulk and the interface. Such a physically accurate approach falls beyond the scope of our work, and it is in fact seldom used by hydrodynamicists. Instead, most studies on soap films use a simplified linear relation between surface tension variations and interfacial elongations (Tambe \& Sharma 1991; Bruinsma et al. 1992; Schwartz \& Roy 1999).

This simplification appears naturally if one considers the surfactants as insoluble, or if their transfer between the bulk and the interface is much slower than any other process. In that case, the coupling with the bulk disappears, and the mass conservation equation for the surfactant becomes at steady state (Park 1991), and in the approximation of quasi-parallel interfaces (see $\S 2.3$ ):

$$
\partial_{x}\left(\Gamma u_{s}\right)-D^{s} \partial_{x x} \Gamma=0,
$$

where $u_{s}$ is the velocity of the interface and $D^{s}$ is the surface diffusion coefficient. If, moreover, the Péclet number $\ell U / D^{s}$ is large (with $\ell$ the characteristic length scale along $x$ ), surface diffusion is negligible and the above equation reduces to $\partial_{x}\left(\Gamma u_{s}\right)=0$. This leads to

$$
\Gamma u_{s}=\Gamma_{0} u_{s}^{*},
$$

where $\Gamma_{0}$ and $u_{s}^{*}$, respectively, are the interfacial surfactant concentration and velocity in the meniscus. Note that this reference value is only well-defined if the interfacial velocity converges towards a finite limit $u_{s}^{*}$ in the meniscus. For small variations of the surface tension around its equilibrium value $\gamma_{0}=\gamma\left(\Gamma_{0}\right)$, we get

$$
\gamma=\gamma_{0}+\partial_{\Gamma} \gamma\left(\Gamma-\Gamma_{0}\right)=\gamma_{0}+\partial_{\Gamma} \gamma \Gamma_{0}\left(u_{s}^{*} / u_{s}-1\right),
$$

where $\partial_{\Gamma}$ is henceforth used to mean $\left.\partial_{\Gamma}\right|_{\Gamma=\Gamma_{0}}$. Defining the surface modulus $E=-\partial_{\Gamma} \gamma \Gamma_{0}(>0)$ and

$$
\sigma=\left(\Gamma_{0}-\Gamma\right) / \Gamma_{0}=1-u_{s}^{*} / u_{s}
$$

we get

$$
\gamma=\gamma_{0}+E \sigma
$$

Equation (2.9) completes our set of equations (2.1)-(2.4).

It is important to keep in mind that slightly different assumptions lead to different definitions of $E$ and $\sigma$, and a different set of equations. The choice of a particular relation is somewhat arbitrary, and we present in appendix B two other widely used definitions for $\sigma$ and $E$ to facilitate comparison of our work to the literature. We checked that, in our particular problem, the different choices lead qualitatively to the same conclusions.

\subsection{Dimensionless equations}

To predict the shape and thickness of the film, we classically consider separately three domains (Levich 1962; Quéré 1999). (i) At the top of the film $(x \rightarrow+\infty)$, there is a 
region of uniform thickness $2 h_{\infty}$, and uniform velocity $U$. (ii) Near the bath, there is the 'static meniscus' of constant radius of curvature $r_{m}$, much thicker than the constant thickness film. This size discrepancy ensures that although the interfacial velocity in this second region can be non-zero, the induced forces and dissipation are negligible, and the meniscus can be considered at rest (whence the name). In particular, its shape is unaffected by the withdrawal. (iii) The 'dynamic meniscus' bridges the first two domains. Its shape and velocity field are non-trivial, and are computed using the lubrication equations established below. Its extension in the $x$-direction is denoted by $\ell$. An asymptotic matching to the flat film $\left(h(x \rightarrow+\infty)=h_{\infty}\right)$ and to the static meniscus (radius of curvature $r_{m}$ for $x \rightarrow-\infty$ ) serves as boundary conditions to determine the shape of the dynamic meniscus, and sets the value of $h_{\infty}$.

The two length scales $h_{\infty}$ and $\ell$ are used to non-dimensionalize the variables. They are both a priori unknown, but we assume that they satisfy $h_{\infty} \ll \ell$, a very classical approximation for this kind of problems. Hence all equations will be solved below at the lowest sensible order in the small parameter $\varepsilon=h_{\infty} / \ell$. We set $x=\ell X, y=\varepsilon \ell Y$, $h=\varepsilon \ell H, v_{x}=U V_{X}, v_{y}=\varepsilon U V_{Y}, q=Q \varepsilon U \ell, p=(\eta U / \ell) P, \gamma=\gamma_{0}+E \sigma$.

By definition of the length and velocity scales, $H, V_{x}, V_{y}, Q$ and their derivatives along $X$ remain of order one in the dynamic meniscus region. In contrast, the choice of rescaling $P$ by a typical pressure based on the length $\ell$ (instead of $h_{\infty}$, for example) is arbitrary at this point. Hence, as discussed in detail by Breward \& Howell (2002), the order of $P$ is not constrained.

Three dimensionless numbers describe the system. The capillary number $C a=$ $\eta U / \gamma_{0}$ compares viscous and capillary forces. The Marangoni number $M a=E /(\eta U)$ compares interface elasticity to viscous forces. The Boussinesq number $B q=\eta_{s}^{*} /(\eta \ell)$ compares surface and bulk viscosities.

Rescaling equations (2.1) and (2.2), we get

$$
\begin{gathered}
\partial_{Y Y} V_{X}+\varepsilon^{2} \partial_{X X} V_{X}-\varepsilon^{2} \partial_{X} P=0, \\
\partial_{Y Y} V_{Y}+\varepsilon^{2} \partial_{X X} V_{Y}-\partial_{Y} P=0, \\
\partial_{X} V_{X}+\partial_{Y} V_{Y}=0
\end{gathered}
$$

The explicit expressions (2.3) and (2.4) of the stress continuity at the interface are complex (see appendix C). Below we only write their simplified form, where each term has been evaluated at the lowest order in $\varepsilon$. Setting $V_{s}(X)=V_{X}(X, H(X))$, we obtain

$$
\begin{gathered}
-P-2 V_{s}^{\prime}=\varepsilon H^{\prime \prime}\left(C a^{-1}+M a \sigma+B q V_{s}^{\prime}\right)=\varepsilon H^{\prime \prime}\left(C a^{-1}+\Xi\right), \\
\partial_{Y} V_{X}+\varepsilon^{2}\left(\partial_{X} V_{Y}-2 H^{\prime 2} \partial_{Y} V_{X}+4 H^{\prime} \partial_{Y} V_{Y}\right)=\varepsilon B q V_{s}^{\prime \prime}+\varepsilon M a \sigma^{\prime}=\varepsilon \Xi^{\prime},
\end{gathered}
$$

where we have introduced the dimensionless dynamical stress at the interface $\Xi(X)=M a \sigma+B q V_{s}^{\prime}$ satisfying $\tau_{s}=\eta U\left(C a^{-1}+\Xi\right)$. The prime notation indicates the derivative for functions depending only on $X$.

Lastly, mass conservation is given by $Q=1$. In the rest of this paper, we will focus on solutions of this set of equations.

\subsection{Thin film approximation: lubrication and elongational flows}

The next step in the resolution of our problem is to compare the different terms of equations (2.10)-(2.14) to simplify them. This step is surprisingly subtle, since, as already stressed, the order of magnitude of the rescaled pressure $P$ is not known a priori (Breward \& Howell 2002) and thus cannot be compared to the other terms. And in fact, two mutually exclusive cases must be considered: the lubrication 
case with a viscous stress dominated by the Poiseuille flow in the film, and the elongational case with a constant velocity across the film (plug flow). Each of these cases corresponds to a different scaling for $P$.

Problems with a no-slip condition on one side of the thin film, such as the Landau-Levich-Derjaguin (LLD) problem (Landau \& Levich 1942; Derjaguin 1943), or on both sides, such as Reynolds drainage (Reynolds 1886), fall within the lubrication case. Indeed, these boundary conditions impose that $\partial_{Y Y} V_{X}$ should be of order one (and non-zero). Since $\partial_{X X} V_{X}$ is also of order one, (2.10) reduces to $\partial_{Y Y} V_{X}=\varepsilon^{2} \partial_{X} P$. Consistently, the pressure $P$ must be of order $\varepsilon^{-2}$ : in physical units this corresponds to $p \sim \eta U \ell / h_{\infty}^{2}$, in agreement with Poiseuille flow.

The other limit is observed for interfaces with vanishing tangential stresses, such as viscous sheets or threads of pure Newtonian liquids (Wilson 1988; Gaudet et al. 1996). In that case $\partial_{Y} V_{X}$ vanishes at the interface, and $\partial_{Y Y} V_{X} \ll 1$. The dimensionless pressure is in that case of order one, so $p \sim \eta U / \ell$, in agreement with an elongational flow.

The external conditions often determine the relevant case. For soap films, however, the tangential stress is generated by surfactant transport, which is coupled to the flow in the film. Depending on the geometry and the scale (both spatial and temporal) of the problem, and the physical and thermodynamical properties of the surfactants (diffusion and adsorption constants, for example), one may a priori expect either one of these limits. And in fact, in the literature both cases have been investigated (Mysels et al. 1959; de Gennes 2001; Howell \& Stone 2005; van Nierop et al. 2008).

In the situation that we consider (a film pulled from a bath, at steady state, with viscous or elastic interfacial rheology) we show that only the lubrication assumption, which is investigated below, leads to a self-consistent solution. The elongational case is discussed and discarded in $\S 5$.

\section{Lubrication flow}

As we just discussed, what we call the 'lubrication case' corresponds to a pressure $P$ of order $\varepsilon^{-2}$ with our choice of rescaling. Equation (2.11) thus imposes at lowest order $\partial_{Y} P=0$, which allows us to integrate (2.10). Using $\partial_{Y} V_{X}(X, 0)=0$, we obtain

$$
V_{X}(X, Y)=\varepsilon^{2} P^{\prime}(X)\left(Y^{2} / 2-H^{2} / 2\right)+V_{s}(X) .
$$

The flow in the film is thus a simple Poiseuille flow.

The tangential stress continuity (2.14) leads to

$$
\partial_{Y} V_{X}=\varepsilon B q V_{s}^{\prime \prime}+\varepsilon M a \sigma^{\prime},
$$

for $Y=H$, where $\partial_{Y} V_{X}$ has been assumed of order one. This implies that $\left(M a \sigma+B q V_{s}^{\prime}\right)^{\prime}$ is of order $\varepsilon^{-1}$, as well as $M a \sigma+B q V_{s}^{\prime}$. In (2.13), the only term able to compensate the pressure term of order $\varepsilon^{-2}$ is thus $\varepsilon H^{\prime \prime} / C a$. This implies that the aspect ratio $\varepsilon$ is of the order of $\mathrm{Ca}^{1 / 3}$ and $\ell \sim h_{\infty} \mathrm{Ca}^{-1 / 3}$.

Equation (2.13) becomes

$$
P=-\frac{\varepsilon}{C a} H^{\prime \prime}
$$

Hence, by integration of (3.1),

$$
Q=\frac{\varepsilon^{3}}{3 C a} H^{3} H^{\prime \prime \prime}+V_{s} H=1 .
$$


Another equation is obtained from (3.2), which involves the interfacial rheological properties. Using (3.1) and (3.3), this equation becomes

$$
\varepsilon^{2} H H^{\prime \prime \prime}+B q C a V_{s}^{\prime \prime}+M a C a \sigma^{\prime}=0
$$

or, once integrated between $X$ and $+\infty$,

$$
\varepsilon^{2}\left(H H^{\prime \prime}-\frac{1}{2} H^{\prime 2}\right)+B q C a V_{s}^{\prime}+M a C a\left[\sigma(X)-\sigma_{\infty}\right]=0
$$

As discussed in van Nierop et al. (2008), these two equations respectively express the force balance in the $X$-direction written locally and on the upper part of the film (between $X$ and $+\infty$ ), divided by $\gamma$. Note that each term is expressed at its lowest order in $\varepsilon$, independently of the others.

\subsection{Incompressible interface}

For large interfacial viscosity or elasticity, the tangential stress boundary condition at the interface (3.2) reduces to $V_{s}^{\prime}=0$, the condition of interfacial incompressibility. Given the condition $V_{s} \rightarrow 1$ as $X \rightarrow+\infty$, this imposes $V_{s}=1$ everywhere. This is the classical assumption made by Frankel (Mysels et al. 1959). Setting $\varepsilon=(3 C a)^{1 / 3},(3.4)$ becomes

$$
H^{\prime \prime \prime}=\frac{1-H}{H^{3}} .
$$

The film deposited on a solid wall pulled at constant velocity $U$ out of a bath of pure liquid is governed by the same equations. This problem is known as the LLD problem (Landau \& Levich 1942; Derjaguin 1943). The line $y=0$ of the Frankel's film, along which the viscous stress is zero by symmetry, plays the role of the free interface of the LLD film; the incompressible interface of the Frankel's film moving at velocity $U$ plays the role of the solid wall pulling the LLD film.

Equation (3.7) is solved with the following boundary conditions: for $X \rightarrow+\infty$, $H \rightarrow 1, H^{\prime} \rightarrow 0$ and $H^{\prime \prime} \rightarrow 0$. The length scale $h_{\infty}$ (which is the unknown quantity to be computed) is determined by the asymptotic matching of the solution at $X \rightarrow-\infty$ to the static meniscus of radius $r_{m}$. This problem admits a unique solution, for which the thickness at large $x$ is $h_{F r}=2 h_{\infty}=1.28 r_{m}(3 C a)^{2 / 3}$.

One important remark is that an interfacial stress gradient is required to pull the Frankel's film out of the bath. Indeed, as discussed in Cantat (2013), the tangential stress continuity (2.14) integrated along the film requires that

$$
\Xi_{\infty}-\Xi_{-\infty}=\frac{3}{(3 C a)^{1 / 3}} \int_{-\infty}^{\infty} \frac{H-1}{H^{2}} \mathrm{~d} X .
$$

The numerical value of the last integral is 1.85 . Coming back to physical quantities, we get

$$
\delta \tau_{s}=\eta U \delta \Xi=1.85 \gamma(3 C a)^{2 / 3},
$$

where $\delta \tau_{s}$ is the interfacial stress difference between the steady thin film and the meniscus.

A non-zero interfacial extension must occur to produce this interfacial stress variation, by either interfacial elasticity or viscosity. If these extensions are small enough (which is the case for 'large' elasticities or interfacial viscosities), they do not affect the solution, and the assumption of incompressible interface is justified. However, if non-negligible extensions are needed to produce $\delta \tau_{s}$, the interfacial 
rheology must be explicitly taken into account in the model. We will now consider independently these two limiting cases of purely viscous and purely elastic interfaces.

\subsection{Viscous interface}

In the case of purely viscous interfaces $(M a=0)$, with $\varepsilon=(3 C a)^{1 / 3}$, the flux balance (3.4) and the force balance (3.5) lead to a closed system for $H$ and $V_{s}$ :

$$
\begin{gathered}
V_{s}^{\prime \prime}=\lambda_{v i s} \frac{V_{s} H-1}{H^{2}}, \\
H^{\prime \prime \prime}=\frac{1-V_{s} H}{H^{3}},
\end{gathered}
$$

with $\lambda_{\text {vis }}=3^{2 / 3} \mathrm{~Bq}^{-1} \mathrm{Ca}^{-1 / 3}$. The boundary conditions for $H$ are unchanged from the incompressible case, and in particular the matching to the static meniscus imposes that $H^{\prime \prime}$ tends to a finite positive value $H_{\text {out }}^{\prime \prime}$ at $X \rightarrow-\infty$. The interfacial velocity satisfies $V_{s} \rightarrow 1$ and $V_{s}^{\prime} \rightarrow 0$ for $X \rightarrow+\infty$, and must also reach a finite value in the meniscus region $(X \rightarrow-\infty)$ to be matched to the outer problem.

The equation set solved with boundary conditions at large $X$ admits a solution. However, the velocity diverges in the meniscus and the solution is thus non-physical. This means that a steady state film of constant thickness cannot exist with a simple viscous interface. This important result can be physically pictured by considering the fact that a force must arise to extract the film out of the reservoir. Capillary and bulk viscous forces resist the extraction, thus the only driving force is due to interfacial viscosity. At steady state, a constant traction, hence a constant and non-zero velocity gradient along the interface, must appear in the film: this is in contradiction to the conditions of constant interfacial velocity and constant thickness in the steady thin film.

One way to prove this incompatibility in a more formal and rigorous way is to consider an energy balance on the dynamic meniscus. We show in appendix D that the solution of equations (3.10) and (3.11) diverges at least like $|x|^{1 / 2}$ in the meniscus.

\subsection{Elastic interface}

We now consider a purely elastic interface, by setting $B q=0$ in our system (3.4)-(3.5). The fundamental difference to the viscous case lies in the fact that the surface stresses now only depend on the (Lagrangian) extension of a portion of the interface, and not on its velocity. They may persist in the flat film region, and in fact we find this rheology to be compatible with the pulling of a steady film.

Using (2.8) with $u_{s}^{*} / U=V_{s}^{*}$, the local force balance equation (3.5) becomes

$$
\varepsilon^{2} H H^{\prime \prime \prime}+C a M a \frac{V_{s}^{*}}{V_{s}^{2}} V_{s}^{\prime}=0,
$$

and a closed set of equations is given by (3.4) and (3.12). With $\varepsilon=(3 C a)^{1 / 3}$, we get the final form of our system of equations:

$$
\begin{gathered}
V_{s}^{\prime}=-\frac{\lambda V_{s}^{2}\left(1-V_{s} H\right)}{H^{2}}, \\
H^{\prime \prime \prime}=\frac{1-V_{s} H}{H^{3}},
\end{gathered}
$$

with $\lambda=\lambda_{0} / V_{s}^{*}$ and $\lambda_{0}=(3 C a)^{2 / 3}(\gamma / E)$. Here $\lambda$ is the only parameter of our system, and it compares interfacial elasticity to surface tension. The matching conditions are 


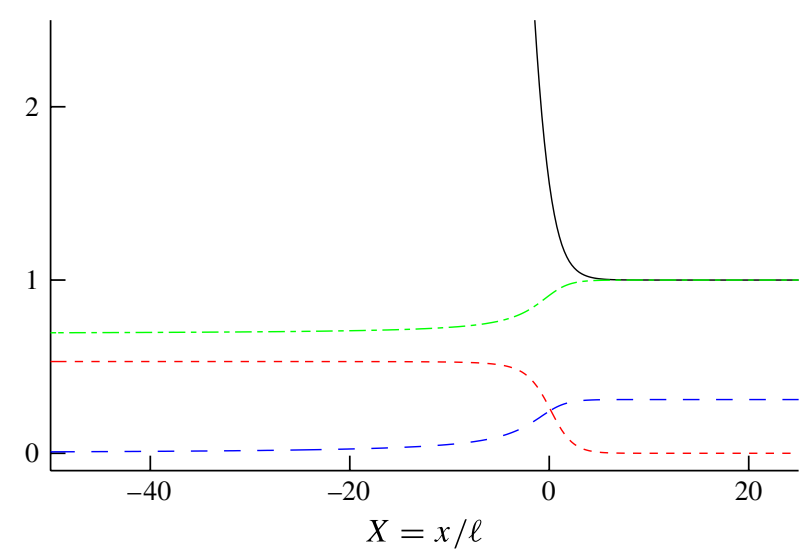

Figure 2. (Colour online) Characteristic solution of the system (3.13)-(3.14) for $\lambda=0.28$. Solid black line, $H(X)$; short-dashed red line, $H^{\prime \prime}(X)$; dot-dashed green line, $V_{s}(X)$; longdashed blue line, $\sigma(X)$. As expected, the film reaches a constant thickness $(H(X \rightarrow+\infty)=1)$ towards the flat film region, and a constant curvature towards the static meniscus. For this particular value of $\lambda, H^{\prime \prime}(X \rightarrow-\infty)=0.53$, which is slightly lower than what is obtained in Frankel's theory (0.64): this means that the extracted film is slightly thinner than for perfectly incompressible interfaces.

unchanged: for $X \rightarrow+\infty, H=1, H^{\prime}=H^{\prime \prime}=0, V_{s}=1$, and the length scale $h_{\infty}$ is determined from the curvature condition at $X \rightarrow-\infty$.

Incompressibility of the interface corresponds to 'large' elasticities, that is, $\lambda \ll 1$. In this limit, (3.13) simply predicts a constant interfacial velocity $u_{s}=U$, and Frankel's theory is recovered. We now solve our system (3.13)-(3.14) for a large range of $\lambda$ and discuss its solutions.

\section{Numerical results for elastic interfaces}

\subsection{Numerical resolution}

The boundary conditions are specified at $X \rightarrow+\infty$. To incorporate them into our numerical resolution, we linearize the system (3.13)-(3.14) around $H=1, V_{s}=1$, and solve it analytically between $X=X_{0}$ and $X \rightarrow+\infty$. The arbitrary choice of $X_{0}$ allows us to impose, without loss of generality, $H\left(X_{0}\right)=1+\varepsilon_{H}$, with $\varepsilon_{H} \ll 1$. Setting $H^{\text {lin }}=1+\varepsilon_{H} \exp \left[\mu\left(X-X_{0}\right)\right]$ and $V_{s}^{\text {lin }}=1+\alpha \varepsilon_{H} \exp \left[\mu\left(X-X_{0}\right)\right]$, we obtain the two conditions $\alpha \mu=\lambda(1+\alpha)$ and $\mu^{3}=-1-\alpha$. This leads to $\alpha=1 /(\mu / \lambda-1)$ and $\mu^{3}-\lambda \mu^{2}+1=0$. The function $\mu \mapsto \mu^{3}-\lambda \mu^{2}+1$ is increasing in the range of negative values of $\mu$, and equals 1 for $\mu=0$, hence it has exactly one real negative root. Moreover, the sum of its three roots equals $\lambda>0$. Hence, the two other roots of $f$ are either both real positive, or complex conjugates with a positive real part. Hence, this third-order equation has only one root $\mu$ with a negative real part, and a unique solution is thus obtained for each value of $\lambda$.

This solution $\left(H^{\text {lin }}, V_{s}^{\text {lin }}\right)$ of the linear problem is used as the boundary condition for the numerical resolution of the nonlinear problem. The resolution is performed with MATLAB, and convergence has been obtained for all $\lambda$ in the range $\left[10^{-4}-20\right]$. In all cases, as seen in figure $2, H^{\prime \prime}$ and $V_{s}$ reach a constant value for large negative values of $X$. The origin of the $X$-axis has been chosen so that the curvature reaches half its maximal value at $X=0$. 

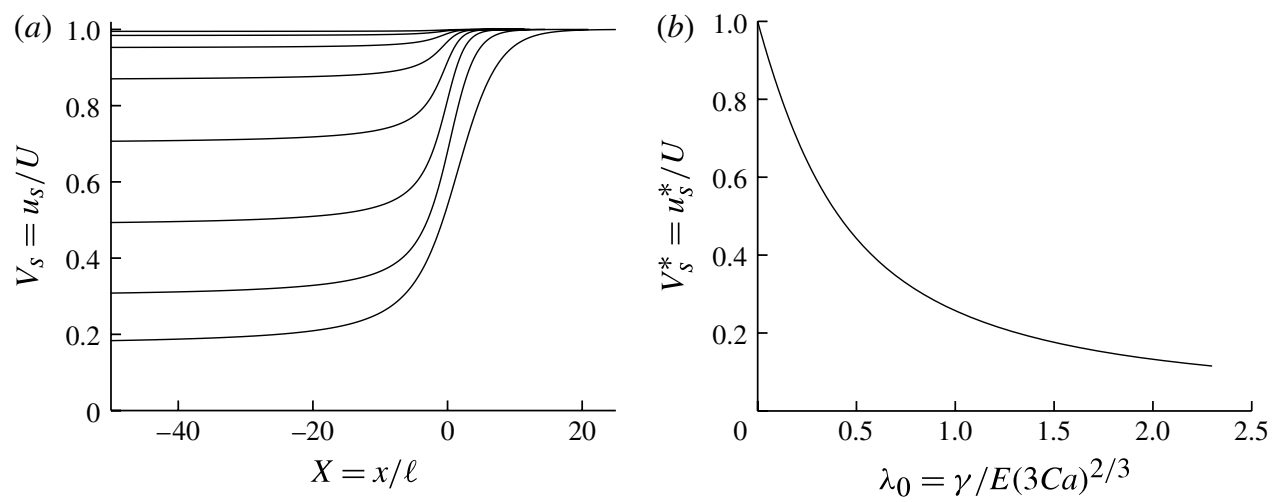

FIGURE 3. (a) Interfacial velocity $u_{s} / U$ in the dynamic meniscus, as a function of $x / \ell$, for different values of $\lambda$. From top to bottom, $\lambda=2.8 \times 10^{-3} ; 8.9 \times 10^{-3} ; 2.8 \times 10^{-2} ; 8.9 \times$ $10^{-2} ; 2.8 \times 10^{-1} ; 8.9 \times 10^{-1} ; 2.8 ; 8.9$. Frankel's assumption of a constant interfacial velocity is recovered when $\lambda$ vanishes (e.g. for diverging interfacial elasticities $E$ ). For larger values of $\lambda, u_{s}$ varies along the film (signature of interface stretching), and reaches a constant value $u_{s}^{*}$ in the meniscus. (b) Asymptotic velocity $u_{s}^{*}$ in the meniscus rescaled by the imposed velocity $U$, as a function of $\lambda_{0}=\gamma / E(3 C a)^{2 / 3}$.

All physical quantities are deduced from the family of solutions $H(X, \lambda), V_{s}(X, \lambda)$. In particular, the length scales $h_{\infty}$ and $\ell=h_{\infty} / \varepsilon=h_{\infty}(3 \mathrm{Ca})^{-1 / 3}$ are deduced by matching the asymptotic value of the curvature of the film to the (static) curvature of the meniscus: $h_{\infty}(\lambda)=r_{m}(3 C a)^{2 / 3} H^{\prime \prime}(-\infty, \lambda)$.

\subsection{Film thickness and velocity field}

Two physical quantities are of particular interest in this problem. The first one is the interfacial velocity $V_{s}=u_{s} / U$, which we plotted in figure $3(a)$ as a function of $X$, for various values of $\lambda=(3 C a)^{2 / 3} \gamma /\left(E V_{s}^{*}\right)$. As anticipated, $V_{s}$ tends towards a well-defined constant value $V_{s}^{*}$ in the meniscus region. This quantity is important to determine. Indeed, the physical control parameter of our problem $\lambda_{0}=(3 C a)^{2 / 3}(\gamma / E)$ differs from the numerical control parameter $\lambda=\lambda_{0} / V_{s}^{*}$ by a factor of $V_{s}^{*}$, which is not known a priori. Comparing these numerical results to experiments would thus require us to determine a posteriori the value of $\lambda_{0}$ corresponding to each numerical parameter $\lambda$.

The parameter $V_{s}^{*}$, shown in figure $3(b)$ as a function of $\lambda_{0}$, has a strong physical meaning, since it is directly related to interface deformation (see (B 2)). A vanishing value of $\lambda_{0}$ corresponds to a diverging elastic modulus and thus an incompressible interface, and accordingly the interfacial velocity remains constant along the entire interface in this limit: $V_{s}(X)=V_{s}^{*}=1 . V_{s}^{*}$ then decreases with $\lambda_{0}$, which denotes increasing interfacial stretching in the dynamic meniscus region.

The other important quantity is the film thickness $2 h_{\infty}$, because it is the easiest and most natural quantity to measure in experiments. Its value, rescaled by the Frankel's thickness $h_{F r}=2.68 r_{m} C a^{2 / 3}$, is plotted in figure 4(a) as a function of $\lambda_{0}$. Similarly to what happens for $V_{s}^{*}$, Frankel's theory is recovered for vanishing values of $\lambda_{0}$; then $2 h_{\infty} / h_{F r}$ decreases monotonically as $\lambda_{0}$ increases.

The same data are also plotted in a more familiar fashion in figure $4(b)$ as a function of the capillary number, for various ratios $E / \gamma$, thus allowing for comparison with experimental data. At small capillary numbers, the value of $\lambda_{0}$ is small and 

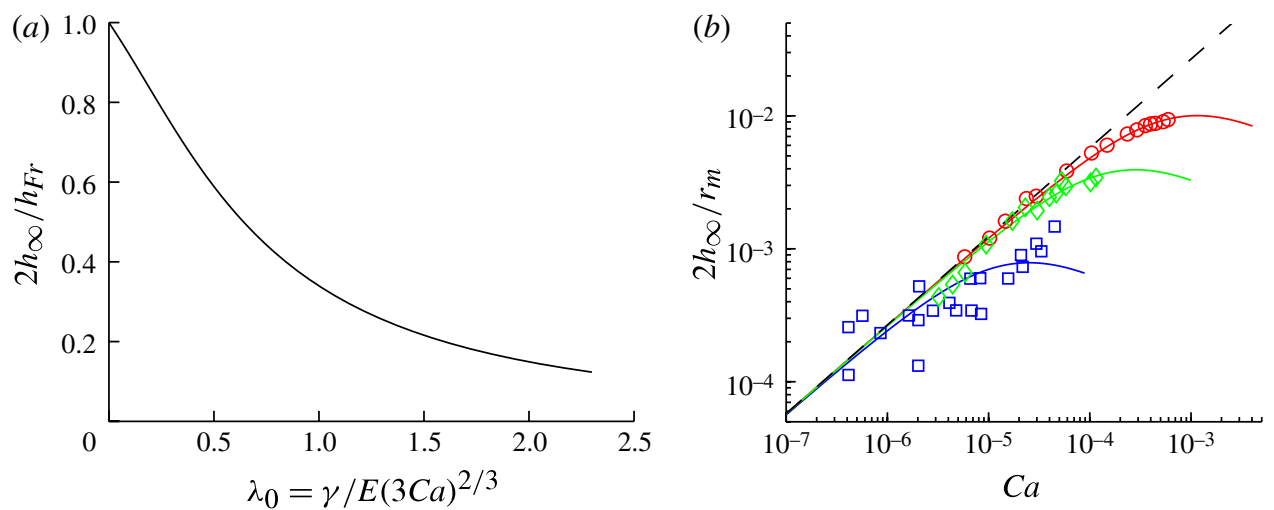

FIgURE 4. (Colour online) (a) Thickness of the film rescaled by the Frankel's thickness, as a function of $\lambda_{0}$. Frankel's law is obeyed at small $\lambda_{0}$ (high elasticities, or low capillary numbers), but the film becomes increasingly thinner as $\lambda_{0}$ increases. (b) Symbols: experimental data obtained respectively with a solution of decanoic acid, concentration $4 \%$, $\mathrm{pH}=11.55$ by Lal \& di Meglio (1994) $(\square)$; a solution of $\mathrm{C}_{12} \mathrm{E}_{5}$ at $0.5 \%$ by Cohen-Addad \& di Meglio (1994) $(\diamond)$; a solution of $\mathrm{C}_{12} \mathrm{E}_{6}$ at $5 \mathrm{cmc}$ by Saulnier et al. (2011) ( $\left.\bigcirc\right)$. Solid lines, the same data as in figure $4(a)$, plotted as a function of the capillary number, for $E / \gamma=0.0018,0.009$ and 0.023 (bottom to top). Dashed line, Frankel's law.

Frankel's law is obeyed. Above a critical capillary number $C a^{c}$, scaling as $(E / \gamma)^{3 / 2}$, the film thickness departs from Frankel's law, as already deduced from scaling analysis in Lal \& di Meglio (1994). The experimental data reported in Cohen-Addad \& di Meglio (1994), Lal \& di Meglio (1994) and Saulnier et al. (2011) are compatible with our theoretical results, as shown in figure $4(b)$. This allows us to estimate the elastic modulus of the different solutions used. The best fit was obtained for $E / \gamma=(0.0018,0.009,0.023)$, and thus $E=(0.066,0.27,0.8) \mathrm{mN} \mathrm{m}^{-1}$ respectively for the solutions of decanoic acid, $\mathrm{C}_{12} \mathrm{E}_{5}$ and $\mathrm{C}_{12} \mathrm{E}_{6}$. This is in agreement with the estimation made in Lal \& di Meglio (1994) for the first solution. These values are too small to be compared to surface moduli obtained by pendant drop methods. Moreover, the elastic moduli deduced from these data are presumably effective moduli, which implicitly take into account the surfactant exchange with the bulk and the diffusion in the film, and which may thus depend on the confinement. Experimentally, the film thickness always remains smaller than the diameter of the frame pulling the film, which also induces a film thickness saturation at high velocities (Lal \& di Meglio 1994). However, this reason can be discarded for the data plotted in figure 4(b), as the saturation occurs for thicknesses significantly smaller than the frame diameter.

Such a transition at a critical capillary number is reminiscent of the results obtained in a Landau-Levich geometry. Models assuming elastic (Park 1991) or viscous (Scheid et al. 2010) interfaces both predict an incompressible behaviour of the interfaces at low velocities and a transition towards another regime above a critical capillary number. However, the nature of these transitions is different from the results plotted in figure $4(b)$. In the Landau-Levich geometry, for viscous and elastic models, the thickness obeys $h_{L L}=1.34 r_{m} \mathrm{Ca}^{2 / 3}$ below the transition and $h_{L L}=3.38 r_{m} \mathrm{Ca}^{2 / 3}$ above, and keeps increasing with $\mathrm{Ca}$ at the transition. In contrast, for soap films, the elastic model predicts that the thickness saturates above the critical capillary number and the viscous model does not admit any steady solution. This underlines a major difference between these two similar problems: the stress-free limit for the liquid/gas interface 


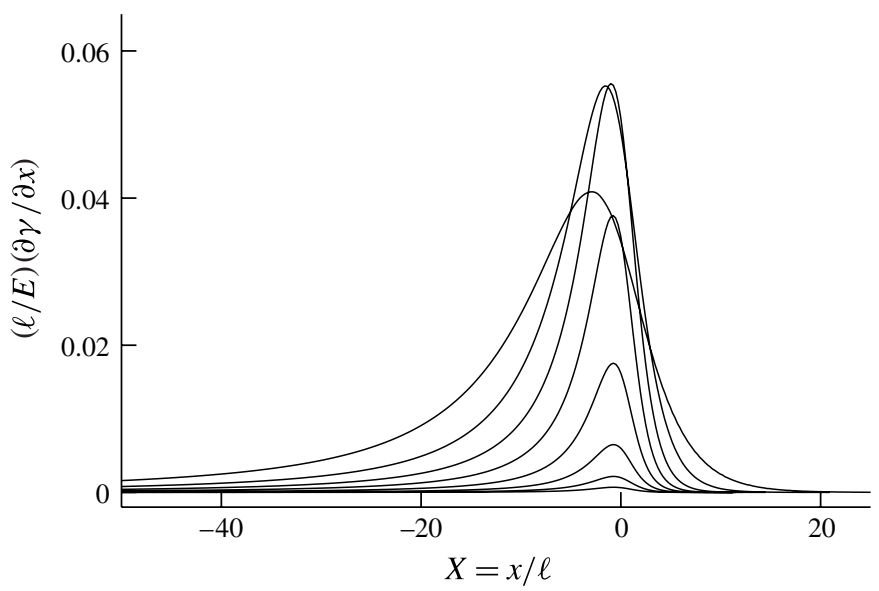

FIGURE 5. Derivative of the tangential stress $\partial_{x} \gamma$ in the dynamic meniscus (rescaled by $E / \ell$ ) along the film, for different values of $\lambda$. From bottom to top (far from the maximum), $\lambda=2.8 \times 10^{-3} ; 8.9 \times 10^{-3} ; 2.8 \times 10^{-2} ; 8.9 \times 10^{-2} ; 2.8 \times 10^{-1} ; 8.9 \times 10^{-1} ; 2.8 ; 8.9$. The stress is constant in the flat film and in the meniscus regions, and varies only in a confined region in the dynamic meniscus (see figure 2 for comparison).

is well defined in the Landau-Levich geometry but is irrelevant for soap films. This comes from the fact that the soap film is pulled out of the liquid bath by the interfaces themselves, which consequently need to be able to generate forces.

The lubrication assumption made to solve the system can now be tested a posteriori with these numerical solutions. In particular, the viscous force associated with elongational flows was assumed to be negligible compared to the Laplace pressure and surface tension forces. At large $\lambda$ the extensional deformation of the film becomes important, and this assumption needs to be checked. The force (acting on the film between $X$ and $+\infty$, per unit of length in the $z$-direction) due to Laplace pressure and surface tension, once rescaled by $\gamma$, is given by $\varepsilon^{2}\left(H H^{\prime \prime}-H^{\prime 2} / 2\right)$ (see (3.6)). In

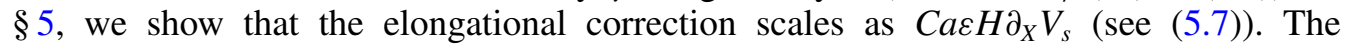
important point is that despite the divergence of $H$ as $X^{2}$, both $H \partial_{X} V_{s}$ and $H H^{\prime \prime}-H^{\prime 2} / 2$ remain of order one for all $X$. Here $\varepsilon$ is of the order of $\mathrm{Ca}^{1 / 3}$ and the elongational force is negligible as long as $C a^{2 / 3} \ll 1$. The numerical solution is thus self-consistent, independently from the value of $\lambda_{0}$.

\subsection{Interfacial stress}

To better understand the physics of the thinning, it is important to examine the interfacial stress $\tau_{s}$. In our purely elastic model, $\tau_{s}$ is simply the surface tension $\gamma(x)=\gamma_{0}+E\left[1-u_{s}^{*} / u_{s}(x)\right]$, which is directly related to interfacial velocity $u_{s}$. In the meniscus region, the latter is constant $\left(u_{s}=u_{s}^{*}\right)$, hence $\gamma$ is constant and equal to the equilibrium surface tension $\gamma_{0}$. In the flat film region, $u_{s}=U$ and $\gamma$ reaches another constant, larger than $\gamma_{0}$ (since $u_{s}^{*}<1$ ).

As shown in figure 5, the variation of $\gamma$ is localized in the transition region between the flat film and static meniscus, where the curvature of the interface changes rapidly (see figure 2 for comparison).

The driving force pulling the film out of the bath is directly given by the surface tension difference between the flat film region and the static meniscus, given by $\delta \gamma=E\left(1-u_{s}^{*} / U\right)$. The quantity $\delta \gamma / E$ is plotted in figure $6(a)$ as a function 

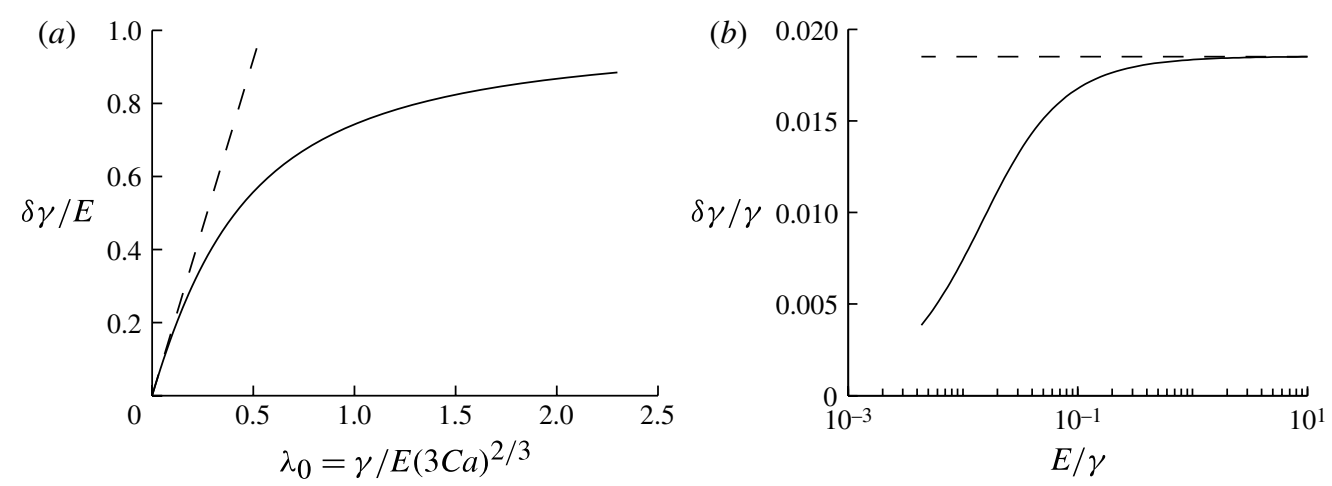

FIGURE 6. Surface tension difference between the thin film and the meniscus $(a)$ rescaled by $E$, plotted as a function of $\lambda_{0}$, and $(b)$ rescaled by $\gamma$, plotted as a function of $E / \gamma$, for $C a=10^{-3}$. In both graphs, the dashed line is Frankel's prediction.

of $\lambda_{0}$. When $\lambda_{0} \ll 1$, a linear variation $\delta \gamma / E \sim 1.85 \lambda_{0}$ is found. This is indeed expected for an incompressible interface limit, in which a surface tension difference $\delta \gamma / \gamma=3.84 C a^{2 / 3}$ is predicted (see (3.9)).

Figure $6(b)$ shows the surface tension difference as a function of the elastic modulus, at constant capillary number. The curve increases monotonically: the driving force generated by the system through interface stretching increases with the surface elasticity. For large values of $E / \gamma, \delta \gamma / \gamma$ reaches a plateau corresponding to the Frankel limit. At lower values of $E / \gamma$, the driving force lowers, and (from the previous section) a thinner film is extracted.

Lastly, from a more experimental standpoint, computing $\delta \gamma$ allows us to determine the interfacial concentration in the extracted flat film $\Gamma_{f}$, which is given by the relation $\left(\Gamma_{0}-\Gamma_{f}\right) / \Gamma_{0}=\delta \gamma / E$ (and which can thus be read on the same graphs). Given a surfactant solution, this information may help to validate the linear elastic model chosen here for the interfacial rheology.

\section{Absence of a steady solution in the elongational limit}

In this last section, we come back to the initial set of equations (2.10)-(2.12) and we investigate another possible limit. So far we have used the lubrication approximation: we simplified (2.10) in $\S 3$ by assuming $\varepsilon^{2} \partial_{X X} V_{X} \ll \partial_{Y Y} V_{X}$, and we checked the self-consistency in $\S 4.2$. The other possibility is to assume that $\varepsilon^{2} \partial_{X X} V_{X}$ is at least of the same order as $\partial_{Y Y} V_{X}$. This leads to an elongational flow in the film, and we show that it does not produce an acceptable solution.

\subsection{Governing equations in the elongational limit}

We derive the new set of simplified equations in the same way as Breward (1999) and van Nierop et al. (2008). Since $\partial_{X X} V_{X}$ is of order one, assuming $\partial_{Y Y} V_{X}$ of the order of $\varepsilon^{2} \partial_{X X} V_{X}$ means in a more formal way that $\partial_{Y Y} V_{X}=O\left(\varepsilon^{2}\right)$. Together with (2.10), it also imposes that $P$ is of order $\varepsilon^{0}$ (instead of $\varepsilon^{-2}$ as in the lubrication approximation).

With this information, we now simplify our system at the lowest order in $\varepsilon$. For convenience, we systematically expand each variable $\left(V_{X}, V_{Y}\right.$ and $\left.P\right)$ in order of $\varepsilon$, writing for example $V_{X}=V_{0, X}+\varepsilon V_{1, X}+\varepsilon^{2} V_{2, X}+\cdots$.

The initial assumption thus becomes $\partial_{Y Y} V_{0, X}=\partial_{Y Y} V_{1, X}=0$, and by symmetry of the film we get $\partial_{Y} V_{0, X}=\partial_{Y} V_{1, X}=0$. Integration of (2.12) thus leads to $V_{0, Y}=-Y \partial_{X} V_{0, X}$. 
Finally, (2.11) implies $\partial_{Y} P_{0}=0$. In simple physical terms, velocity gradients across the film are negligible in this limit, and a plug flow is observed.

At the free interface, the normal and tangential stress continuity equations (2.13) and (2.14) become

$$
\begin{gathered}
-P_{0}-2 \partial_{X} V_{0, X}=\varepsilon H^{\prime \prime}\left(C a^{-1}+M a \sigma+B q \partial_{X} V_{0, X}\right) \\
\partial_{Y} V_{2, X}-H \partial_{X X} V_{0, X}-4 H^{\prime} \partial_{X} V_{0, X}=\frac{B q}{\varepsilon} \partial_{X X} V_{0, X}+\frac{M a}{\varepsilon} \sigma_{0}^{\prime}
\end{gathered}
$$

This imposes $C a \sim \varepsilon$, and $B q$ or $M a$ of order $\varepsilon$. The second-order term $\partial_{Y} V_{2, X}$ is deduced from the $x$-component of the Stokes equation (2.10), at order $\varepsilon^{2}$ :

$$
\partial_{Y Y} V_{2, X}+\partial_{X X} V_{0, X}-\partial_{X} P_{0}=0 .
$$

This implies $\partial_{Y} V_{2, X}=Y\left(-\partial_{X X} V_{0, X}+\partial_{X} P_{0}\right)$. We thus get

$$
\begin{gathered}
\partial_{X} P_{0}=-2 \partial_{X X} V_{0, X}-\frac{\varepsilon}{C a} H^{\prime \prime \prime}, \\
H\left(-\partial_{X X} V_{0, X}+\partial_{X} P_{0}\right)-H \partial_{X X} V_{0, X}-4 H^{\prime} \partial_{X} V_{0, X}=\frac{B q}{\varepsilon} \partial_{X X} V_{0, X}+\frac{M a}{\varepsilon} \sigma_{0}^{\prime},
\end{gathered}
$$

which leads to

$$
{ }_{4} C a \partial_{X}\left(H \partial_{X} V_{0, X}\right)+\varepsilon^{2} H H^{\prime \prime \prime}+B q C a \partial_{X X} V_{0, X}+M a C a \sigma_{0}^{\prime}=0
$$

or, after integration,

$$
4 \varepsilon C a H \partial_{X} V_{0, X}+\varepsilon^{2}\left(H H^{\prime \prime}-\frac{H^{2}}{2}\right)+B q C a \partial_{X} V_{0, X}+\operatorname{MaCa}\left(\sigma_{0}-\sigma_{\infty}\right)=0 .
$$

These equations are the same force balances as (3.5) and (3.6), except that the viscous force in the bulk $H \partial_{X} V_{0, X}$ now appears at dominant order.

The problem is closed using the flux conservation written at order $\varepsilon^{0}, Q=V_{0, X} H=$ 1 , which imposes $V_{0, X}=1 / H$. With $\sigma=1-V_{s}^{*} / V_{s}=1-V_{s}^{*} H$ after (2.8), we obtain

$$
4 \frac{H^{\prime}}{H}+\frac{\varepsilon}{C a}\left(\frac{H^{\prime 2}}{2}-H H^{\prime \prime}\right)+\frac{B q}{\varepsilon} \frac{H^{\prime}}{H^{2}}+\frac{M a V_{s}^{*}}{\varepsilon}(H-1)=0 .
$$

\subsection{Asymptotic analysis for the viscous case}

Following the work by van Nierop et al. (2008), it can be shown that (5.8) has no solution for the purely viscous case $(M a=0)$. The simplest way to see it is to multiply (5.8) by $H^{-3 / 2}$ and integrate it with respect to $X$, leading to

$$
\frac{8}{3} H^{-3 / 2}+\frac{\varepsilon}{C a} H^{\prime} H^{-1 / 2}+\frac{2 B q}{5 \varepsilon} H^{-5 / 2}=\frac{8}{3}+\frac{2 B q}{5 \varepsilon}>0 .
$$

No solution of our problem can satisfy the above inequality. Indeed, if $H$ were a solution, the first and third terms of (5.9) would tend towards 0 for $X \rightarrow-\infty$, while the second term would be negative. Therefore, the equation for an elongational motion with viscous interfaces does not admit any steady solution connecting to the bath.

The reason is the same as for the Poiseuille viscous flow: surface viscous forces are negligible in the meniscus and in the asymptotic thin film, and thus no surface viscous force difference can pull a film out of the meniscus. 


\subsection{Asymptotic analysis for the elastic case}

Equation (5.8) is finally tested for purely elastic interfaces, i.e. $B q=0$ and $M a \neq 0$. The equation becomes

$$
\frac{H^{\prime}}{H}+\frac{H^{\prime 2}}{2}-H H^{\prime \prime}+\xi(H-1)=0
$$

if we set $\varepsilon=4 C a$ and $\xi=M a V_{s}^{*} /(16 C a)$.

This last case is more subtle and the previous argument, used for viscous interfaces, does not allow us to conclude. However, we show below that the total flux deduced from a hypothetical solution of equation (5.10) would not be dominated by the first term of its Taylor expansion: the assumptions made would therefore not be selfconsistent, and the elongational solution is not acceptable.

From (5.3), the velocity field obeys

$$
V_{2, X}=\frac{Y^{2}}{2}\left(\partial_{X} P_{0}-\partial_{X X} V_{0, X}\right)+B,
$$

where $B$ is an integration constant. Using (5.4), $\varepsilon=4 C a$ and $V_{0, X}=1 / H$, this becomes

$$
V_{2, X}=\frac{Y^{2}}{2}\left[-3 \partial_{X X}\left(\frac{1}{H}\right)-4 H^{\prime \prime \prime}\right]+B .
$$

Finally, the flux is, at order $\varepsilon^{2}$,

$$
Q=1+\varepsilon^{2} B H+\frac{\varepsilon^{2}}{6}\left(3 H^{\prime \prime} H-6 H^{\prime 2}-4 H^{3} H^{\prime \prime \prime}\right) .
$$

Since $H$ is assumed to be a solution of (5.10), the last term can be transformed into

$$
I=\xi-\xi H-4 \xi H^{2} H^{\prime}-\frac{5}{2} H^{\prime 2}-\frac{H^{\prime}}{H} .
$$

As $H$ behaves asymptotically as $X^{2}$ when $X \rightarrow-\infty$, the term $-4 \xi H^{2} H^{\prime}$ dominates and the whole expression diverges like $X^{5}$. Note that $\xi>0$ as the velocity $V^{*}$ cannot vanish in a steady elastic case.

The flux is then dominated by the Poiseuille term close to the meniscus, and the elongational assumption $V_{0}=1 / H$ is thus not valid.

\section{Conclusion}

In this paper we systematically derive the equations governing the steady film profile obtained by pulling a frame out of a surfactant solution at constant velocity, for elastic or viscous interfaces, and for two important classes of confined flows, governed either by a Poiseuille velocity field or by an elongational velocity field. We show that purely viscous interfaces cannot lead to a steady film. Purely elastic interfaces, in contrast, lead to self-consistent solutions, which are dominated by a Poiseuille flow. The film thickness decreases when the elongational modulus of the interface decreases, which compares favourably with experiments made in a steady regime (Cohen-Addad \& di Meglio 1994; Lal \& di Meglio 1994; Saulnier et al. 2011). Film extraction from menisci also occurs in foams (Biance, Delbos \& Pitois 2011; Seiwert et al. 2013) and plays an important role in foam rheology and stability. Additional studies are required to determine if, in a three-dimensional foam, such film formation can be considered to occur in a quasi-steady regime, in which case our study would be relevant to this topic. 


\section{Acknowledgements}

We thank B. Scheid, E. Rio, H. A. Stone, A.-L. Biance and A. Saint-Jalmes for their constructive remarks. J.S. acknowledges funding from Région Bretagne (CREATE MOUSPORE) and I.C. from the Institut Universitaire de France

\section{Appendix A. Expression of the interfacial stresses}

The surface that we consider is invariant in the $z$-direction and its normal is $\boldsymbol{n}=\left(n_{x}, n_{y}\right)$. In that simple case, the surface projection tensor is

$$
\boldsymbol{I}_{s}=\left(\begin{array}{cc}
n_{y}^{2} & -n_{x} n_{y} \\
-n_{x} n_{y} & n_{x}^{2}
\end{array}\right),
$$

and the velocity gradient tensor $\boldsymbol{D}_{s}$ equals the velocity surface divergence $\left(\boldsymbol{I}_{s}: \boldsymbol{D}_{s}\right) \boldsymbol{I}_{s}$, which simplifies to

$$
\boldsymbol{D}_{s}=\left(\boldsymbol{I}_{s}: \boldsymbol{D}_{s}\right) \boldsymbol{I}_{s}=\left(-n_{x}^{2} \partial_{x} v_{x}-n_{y}^{2} \partial_{y} v_{y}-n_{x} n_{y}\left(\partial_{x} v_{y}+\partial_{y} v_{x}\right)\right) \boldsymbol{I}_{s}=K \boldsymbol{I}_{s},
$$

so

$$
\boldsymbol{\tau}_{s}=\gamma \boldsymbol{I}_{s}+\left(\kappa_{s}-\eta_{s}\right)\left(\boldsymbol{I}_{s}: \boldsymbol{D}_{s}\right) \boldsymbol{I}_{s}+2 \eta_{s} \boldsymbol{D}_{s}=\left(\gamma+\eta_{s}^{*} K\right) \boldsymbol{I}_{s} .
$$

\section{Appendix B. Alternative definitions for $\sigma$ and $E$}

In this article, we have assumed $E=-\Gamma_{0} \partial_{\Gamma} \gamma$ and $\sigma=1-u_{s}^{*} / u_{s}$. These expressions for $E$ and $\sigma$ are generalized from the case of insoluble surfactants, yet we still assume small relative variations of $\Gamma$. However, slightly different assumptions lead to different definitions of $E$ and $\sigma$. We present here two of the most widely used alternatives.

The first alternative is still based on the assumption of a non-diffusive insoluble surfactant, but linearizes the surface tension with respect to the surface area instead of the surface concentration $\Gamma$. Following an infinitesimal portion of interface along its motion from the meniscus at $t=0$ to its position $x$ at time $t$, we define its respective areas $A_{0}$ and $A(x, t)$.

An alternative to the elastic law (2.7) is to assume that

$$
\gamma=\gamma_{0}+\tilde{E} \frac{A-A_{0}}{A_{0}} .
$$

Mass conservation then imposes $A(x, t) \Gamma(x, t)=A_{0} \Gamma_{0}$. At steady state, this imposes that $A \Gamma$ is uniform along the film, and so

$$
A(x) / A_{0}=\Gamma_{0} / \Gamma(x)=u_{s}(x) / u_{s}^{*},
$$

after (2.6).

A consequence is that $\left(A-A_{0}\right) / A_{0}=\left(\Gamma_{0}-\Gamma\right) / \Gamma$. The two definitions (2.7) and (B 1) are thus equivalent for $\left|\Gamma-\Gamma_{0}\right| \ll \Gamma_{0}$. We checked that both choices give qualitatively similar results.

For soluble surfactants, another important limit is discussed in Couder, Chomaz \& Rabaud (1989). It is strictly valid only if the surfactants diffuse across the thin film and adsorb at the interfaces on a negligible time scale. The bulk concentration of surfactants $c$ thus only depends on $x$ and it is in equilibrium with $\Gamma(x)$. Below the critical micelle concentration (CMC), a linear relation may be assumed: $c(x)=\Gamma(x) / h_{c}$, with $h_{c}$ a constant. When the interface is stretched, surfactant adsorption on the newly created interface reduces bulk concentration. 
For purely elongational flows, the velocity is uniform across the film: a material system defined as the portion of film between $x=x_{1}$ and $x=x_{2}$ at time $t$ can be easily tracked, and will still be a portion of the film between two other abscissae $x_{1}^{\prime}$ and $x_{2}^{\prime}$ at a later time.

Surfactant mass conservation on such a closed system imposes

$$
c+\frac{\Gamma}{h}=\Gamma\left(\frac{1}{h_{c}}+\frac{1}{h}\right)=\frac{\Gamma_{0}}{h_{c}},
$$

since in the static meniscus $h \rightarrow \infty$ and $\Gamma=\Gamma_{0}$. Thus

$$
\gamma=\gamma_{0}+\partial_{\Gamma} \gamma\left(\Gamma-\Gamma_{0}\right)=\gamma_{0}-\partial_{\Gamma} \gamma \Gamma_{0} \frac{h_{c}}{h+h_{c}} .
$$

This provides a second alternative constitutive law for $\sigma$. We did not test it on our system, because it has no physical ground when the flow is not strictly elongational (and we have shown that solutions for our problem necessarily feature Poiseuille-type flows).

\section{Appendix C. Boundary conditions for the flow}

The first terms of the $\varepsilon$ expansion of the different terms of (2.3) and (2.4) are derived below. The tangent and normal unit vectors are

$$
\begin{gathered}
\boldsymbol{t}=\left(1-\frac{1}{2} \varepsilon^{2} H^{\prime 2} ; \varepsilon H^{\prime}\right), \\
\boldsymbol{n}=\left(-\varepsilon H^{\prime} ; 1-\frac{1}{2} \varepsilon^{2} H^{\prime 2}\right) .
\end{gathered}
$$

The bulk contribution to the interfacial stress, with $V_{s}(X)=V_{X}(X, H(X))$, is

$$
\begin{gathered}
\frac{\ell}{\eta U} \boldsymbol{n} \cdot \boldsymbol{\tau} \cdot \boldsymbol{n}=-P-2 \partial_{X} V_{X}-2 H^{\prime} \partial_{Y} V_{X}=-P-2 \partial_{X} V_{s}, \\
\frac{\ell}{\eta U} \boldsymbol{t} \cdot \boldsymbol{\tau} \cdot \boldsymbol{n}=\frac{1}{\varepsilon} \partial_{Y} V_{X}+\varepsilon\left(\partial_{X} V_{Y}-2 H^{\prime 2} \partial_{Y} V_{X}+4 H^{\prime} \partial_{Y} V_{Y}\right) .
\end{gathered}
$$

The surface contribution is determined using $\boldsymbol{\tau}_{s}=\tau_{s} \boldsymbol{I}_{s}=\left(\gamma+\eta_{s}^{*} K\right) \boldsymbol{I}_{s}$ and, after (A 2),

$$
\begin{aligned}
\frac{\ell}{U} K=\partial_{X} V_{X}+H^{\prime} \partial_{Y} V_{X}=V_{s}^{\prime}, \\
\ell \nabla_{s} \boldsymbol{I}_{s}=\varepsilon H^{\prime \prime} \boldsymbol{n}, \\
\frac{\ell}{\eta U} \eta_{s}^{*} \nabla_{s} K=B q\left(\partial_{X X} V_{X}+H^{\prime \prime} \partial_{Y} V_{X}-2 H^{\prime} \partial_{Y Y} V_{Y}+H^{\prime 2} \partial_{Y Y} V_{X}\right) \boldsymbol{t}=B q V_{s}^{\prime \prime} \boldsymbol{t}, \\
\frac{\ell}{\eta U} \nabla_{s} \gamma=M a \sigma^{\prime} \boldsymbol{t} .
\end{aligned}
$$

Finally, we get

$$
\begin{gathered}
\frac{\ell}{\eta U} \boldsymbol{n} \cdot \nabla_{s} \boldsymbol{\tau}_{s}=\varepsilon H^{\prime \prime}\left[C a^{-1}+M a \sigma+B q V_{s}^{\prime}\right], \\
\frac{\ell}{\eta U} \boldsymbol{t} \cdot \nabla_{s} \boldsymbol{\tau}_{s}=M a \sigma^{\prime}+B q V_{s}^{\prime \prime},
\end{gathered}
$$

leading to (2.13) and (2.14). 


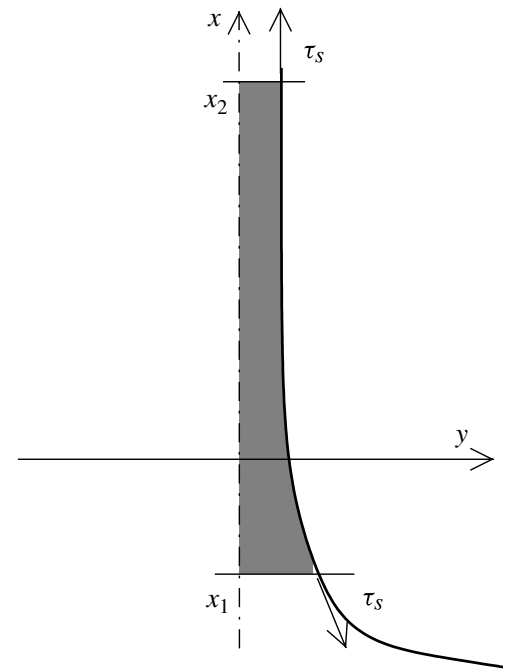

FIGURE 7. System used for the energy balance.

\section{Appendix D. Energy balance on the film, for the lubrication case with viscous interfaces.}

We consider the open system sketched in figure 7 , limited by the symmetry plane of the film, a section of the steady film at position $x_{2}$ and a section of the dynamic meniscus, at position $x_{1}$. The surface free energy of the interface is $\gamma$. In physical units, the surface free energy flux crossing $x_{2}$ is thus $-\gamma u_{s}\left(x_{2}\right)$, with a negative sign indicating energy exiting the open system. This flux exactly compensates the power given by the interface to the system. Finally the pressure is the atmospheric pressure taken as reference pressure, so $p=0$. There is thus no energy exchange across $x_{2}$.

The energy exchanges only come from the meniscus contribution. The power given by the interface is $-\tau_{s} u_{s}\left(x_{1}\right)=-\left(\gamma+\eta_{s}^{*} \partial u_{s} / \partial x\right) u_{s}\left(x_{1}\right)$, partly compensated for by the free energy flux $\gamma u_{s}\left(x_{1}\right)$. The average velocity at $x_{1}$ is $\bar{u}_{1}$, which satisfies the mass balance $h_{\infty} U=h\left(x_{1}\right) \bar{u}_{1}$. The pressure power is $-\gamma h^{\prime \prime} h \bar{u}_{1}=-\gamma h^{\prime \prime} h_{\infty} U$. Finally, in steady state, the energy exchange must compensate the positive energy dissipation in the system and for any $x_{1}$ :

$$
-\eta_{s}^{*} \frac{\partial u_{s}}{\partial x} u_{s}-\gamma h^{\prime \prime} h_{\infty} U>0 .
$$

Now, since $h^{\prime \prime} \rightarrow 1 / r_{m}>0$ as $x \rightarrow-\infty$, we can find a constant $M>0$ and a position $x_{M}$ so that $\gamma h^{\prime \prime}(x) h_{\infty} U>M$ for any $x$ smaller than $x_{M}$. We deduce

$$
-\int_{x}^{x_{M}} \eta_{s}^{*} \frac{\partial u_{s}}{\partial x} u_{s} \mathrm{~d} x^{\prime}>\int_{x}^{x_{M}} M \mathrm{~d} x^{\prime},
$$

leading to $u_{s}(x)>M^{\prime} \sqrt{k-x}$ for any $x$ smaller than $x_{M}$, with $M^{\prime}$ a positive constant and $k$ an arbitrary constant. The velocity is thus diverging in the meniscus. In the frame of a Poiseuille flow assumption, a steady film with purely viscous interfaces can thus not be pulled out of a meniscus. 


\section{REFERENCES}

Alvarez, N. J., Walker, L. M. \& AnNA, S. L. 2012 A criterion to assess the impact of confined volumes on surfactant transport to liquid-fluid interfaces. Soft Matt. 8, 8917-8925.

Berg, S., Adelizzi, E. A. \& Troian, S. M. 2005 Experimental study of entrainment and drainage flows in microscale soap films. Langmuir 21 (9), 3867-3876.

Biance, A.-L., Delbos, A. \& Pitois, O. 2011 How topological rearrangements and liquid fraction control liquid foam stability. Phys. Rev. Lett. 106, 068301.

BREWARD, C. J. W 1999 The mathematics of foam. PhD thesis, Oxford University.

Breward, C. J. W. \& Howell, P. D. 2002 The drainage of a foam lamella. J. Fluid Mech. 458, 379-406.

Bruinsma, R., Di Meglio, J.-M., Quéré, D. \& Cohen-AdDad, S. 1992 Formation of soap films from polymer solutions. Langmuir 8, 3161-3167.

CANTAt, I. 2013 Liquid meniscus friction on a wet wall: bubbles, lamellae and foams. Phys. Fluids 25, 031303.

Cohen-Addad, S. \& Di Meglio, J.-M. 1994 Stabilization of aqueous foam by hydrosoluble polymers. Part 2. Role of polymer/surfactant interactions. Langmuir 10 (3), 773-778.

Couder, Y., Chomaz, J.-M. \& Rabaud, M. 1989 On the hydrodynamics of soap films. Physica D 37, 384-405.

Derjaguin, B. V. 1943 Thickness of liquid layer adhering to walls of vessels on their emptying. Acta Physicochim. USSR 20, 349.

Gaudet, S., McKinley, G. H. \& Stone, H. A. 1996 Extensional deformation of Newtonian liquid bridges. Phys. Fluids 8, 2568-2580.

DE Gennes, P. G. 2001 Young soap films. Langmuir 17 (8), 2416-2419.

Howell, P. D. \& Stone, H. A. 2005 On the absence of marginal pinching in thin free films. Eur. J. Appl. Maths 16, 569-582.

LAL, J. \& Di Meglio, J.-M. 1994 Formation of soap films from insoluble surfactants. J. Colloid Interface Sci. 164 (2), 506-509.

Landau, L. \& Levich, B. 1942 Dragging of a liquid by a moving plate. Acta Physicochim. USSR $17,42$.

LeVich, V. G. 1962 Physicochemical Hydrodynamics. Prentice Hall.

Mysels, K. J., Shinoda, K. \& Frankel, S. 1959 Soap Films: Studies of their Thinning and a Bibliography. Pergamon.

van Nierop, E. A., Scheid, B. \& Stone, H. A. 2008 On the thickness of soap films: an alternative to Frankel's law. J. Fluid Mech. 602, 119-127 and Corrigendum 630, 443 (2009).

PARK, C. W. 1991 Effects of insoluble surfactants on dip coating. J. Colloid Interface Sci. 146 (2), 382-394.

QuÉRÉ, D. 1999 Fluid coating on a fibre. Annu. Rev. Fluid. Mech. 31 (1), 347-384.

REYNOLDS, O. 1886 On the theory of lubrication and its application to Mr. Beauchamp Tower's experiment, including an experimental determination of the viscosity of olive oil. Proc. R. Soc. Lond. 177, 157.

SAGIS, L. M. C. 2011 Dynamic properties of interfaces in soft matter: experiments and theory. Rev. Mod. Phys 83, 1367-1403.

Saulnier, L., Restagno, F., Delacotte, J., Langevin, D. \& Rio, E. 2011 What is the mechanism of soap film entrainment? Langmuir 27 (22), 13406-13409.

Scheid, B., Delacotte, J., Dollet, B., Rio, E., Restagno, F., van Nierop, E. A., Cantat, I., LAngevin, D. \& Stone, H. A. 2010 The role of surface force rheology in liquid film formation. Eur. Phys. Lett. 90, 24002.

SCHWARTZ, L. W \& RoY, R. V 1999 Modelling draining flow in mobile and immobile soap films. J. Colloid Interface Sci. 218 (1), 309-323.

Seiwert, J., Monloubou, M., Dollet, B. \& Cantat, I. 2013 Extension of a suspended soap film: a homogeneous dilatation followed by new film extraction. Phys. Rev. Lett. 111, 094501.

TAmbe, D. E. \& Sharma, M. M. 1991 Hydrodynamics of thin liquid films bounded by viscoelastic interfaces. J. Colloid Interface Sci. 147 (1), 137-151.

Wilson, S. D. R. 1988 The slow dripping of a viscous fluid. J. Fluid Mech. 190, 561-570. 